\title{
The Effect of Temperature on the Spectral Emission of Plasma Induced in Water
}

\author{
B. Charfi \\ Département de Préparation Technologique, Institut Préparatoire aux Etudes d’Ingénieurs de Sfax (IPEIS), 3018 Sfax, Tunisia \\ Correspondence should be addressed to B. Charfi; bilel_charfi@yahoo.com
}

Received 20 June 2012; Revised 25 August 2012; Accepted 4 September 2012

Academic Editor: Gianfranco Giubileo

Copyright (C) 2013 B. Charfi. This is an open access article distributed under the Creative Commons Attribution License, which permits unrestricted use, distribution, and reproduction in any medium, provided the original work is properly cited.

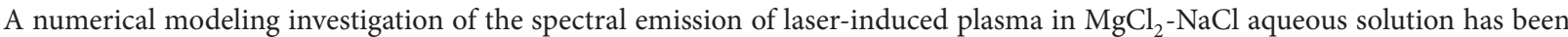
presented. A model based on equilibrium equations has been developed for the computation of the plasma composition and excited levels population. Physical interpretation is presented to comment on firstly the evolution of atomic species number densities, and secondly on the population of the excited species emitting MgII and $\mathrm{NaI}$ resonant lines for temperatures ranging from $3000 \mathrm{~K}$ to $20000 \mathrm{~K}$. It is shown that MgII line reach a maximum of population on the issuing level, at norm temperature of $13800 \mathrm{~K}$. Whereas, $\mathrm{NaI}$ line presents two norm temperatures, evaluated at $3300 \mathrm{~K}$ and $11700 \mathrm{~K}$. This splitting of the NaI norm temperature is explained by the low-ionization potential and weak concentration of the sodium atom in this aqueous solution. Thus, the proposed model can be useful to predict the optimal plasma temperature for the detection of given chemical element, which is not easy to reveal experimentally.

\section{Introduction}

Laser-Induced Breakdown Spectroscopy (LIBS) is identified as the optical emission spectroscopy of the laser-induced plasma [1-3]. LIBS has been acknowledged as a useful tool for the analysis of solid samples. However, for the liquids analysis, the application of such technique was relatively delicate and has been confronted to several drawbacks related to the short lifetime and the poor spectral signal of the plasma induced in liquids [4-8]. In previous work [9], the LIBS sensitivity has been improved by optimization of parameters influencing the spectral emission of the plasma induced on water target. This enhancement has been carried out in a $50 \mathrm{mg} / \mathrm{L} \mathrm{MgCl} 2-\mathrm{NaCl}$ mixed solution; particular attention is paid to the spectral emission of $\mathrm{MgII}(279.55 \mathrm{~nm})$ and $\mathrm{NaI}$ $(588.99 \mathrm{~nm})$ lines. The intensity of those resonant spectral lines, emitted from the laser-induced plasma on the water sample, is found to be dependent on parameters of LIBS analysis system, such as laser pulse energy and delay time (observing time after firing the laser) [9].

Several studies have been reported on the correlation between the LIBS emission spectra from a water target and LIBS system parameters [5, 9-11]. For example, Adamson et al. [10] observed the dependence of LIBS emission spectra, mainly the hydrogen $\mathrm{H}$-alpha and $\mathrm{H}$-beta emission lines on gate time and laser pulse energy.

Samek et al. [5] reported the effect of delay time on the intensities of the $\mathrm{H}$-alpha, $\mathrm{Li}$, and $\mathrm{Na}$ peaks lines when performing LIBS on aqueous samples.

However, relatively few works have been concerned with theoretical approach of the dependence of line intensities emitted from water plasma on the LIBS system parameters; for example, Ahmed and Jaïdane [11] simulated the time evolution of lines intensities emitted from $\mathrm{Ca}, \mathrm{Ca}^{+}, \mathrm{Mg}$, and $\mathrm{Mg}^{+}$using a simple theoretical approach based on electronion recombination. It should be noted that this dependence of lines intensities on the mentioned LIBS system parameters is related, for the most part, to the plasma temperature, since the change of any of the LIBS parameters leads to variation of the plasma temperature.

The work described in this paper is motivated by the desire to gain fundamental knowledge concerning the effect of plasma temperature on Laser-Induced Plasma Spectral Emission (LIPSE), and ultimately to determine the suitable plasma temperature for the detection of a given chemical element. 
The first part of this work is devoted to a brief description of assumptions used for the LIPSE study. The second part presents the calculation of the number densities of species considered in the $\mathrm{MgCl}_{2}-\mathrm{NaCl}$ aqueous solution. In the last part of the paper, results concerning the densities of species emitting MgII and $\mathrm{NaI}$ lines are presented as a function of plasma temperature.

\section{Theoretical}

2.1. Assumptions. The interpretation of LIPSE is a complex task with many unsolved problems. The most important questions concern the ablation mechanisms [12], the chemical reactivity $[13,14]$, the deviations from equilibrium $[12$, $14,15]$, self-absorption phenomenon $[11,15]$, and the matrix effect $[12,16]$.

Nevertheless, the description of LIPSE phenomenon can be simplified by making certain hypothesis $[17,18]$. For this work the following assumptions have to be considered.

(1) Conventionally, LIBS measurements are made in the microsecond time scale, that is, when the LIBS plasma is under recombination conditions. In the microsecond regime of the plasma lifetime, typical values of electron number density $n_{e}$, and plasma temperature $T$, range in the interval $10^{15}<n_{e}<$ $10^{18} \mathrm{~cm}^{-3}$ and $3000<T<20000 \mathrm{~K}[10,14,15]$. The study presented in this paper is limited to this temperature range $3000-20000 \mathrm{~K}$.

(2) The reported $n_{e}$ and $T$ ranges are considered sufficient to create plasma satisfying LTE (Local Thermodynamic Equilibrium) conditions, [10, 14, 15, 18, 19]. Under this LTE hypothesis, the plasma is considered to be sufficiently dilute that particles are independent and indistinguishable, allowing a Boltzmann statistical description and the application of Dalton's law for partial pressures $[16,18-20]$.

(3) For the temperature range considered in this study. the plasma induced in $\mathrm{MgCl}_{2}-\mathrm{NaCl}$ solution, is supposed to be composed of the following species: electrons $\left(\mathrm{e}^{-}\right), \mathrm{H}, \mathrm{H}^{+}, \mathrm{O}, \mathrm{O}^{+}, \mathrm{Na}, \mathrm{Na}^{+}, \mathrm{Mg}, \mathrm{Mg}^{+}$, $\mathrm{Mg}^{++}, \mathrm{Cl}, \mathrm{Cl}^{+}, \mathrm{H}_{2} \mathrm{O}, \mathrm{O}_{2}, \mathrm{OH}$, and $\mathrm{H}_{2}$. Species with elevated ionization energy $\left(E_{i}>20 \mathrm{eV}\right)$ have not been taken into account; indeed, their existence require excessively high temperatures (out of our range of interest).

(4) The laser-induced plasma is supposed to be homogeneous. It is well known that plasmas present a spatial distribution of their parameters. This plasma inhomogeneity yields to a self absorption of resonant spectral lines. Solving a model for those inhomogeneous plasmas could be very complicated. However for a water-plasma at the end of its cooling process and within a short observation window, spatial gradients have a lower influence on the plasma parameters. Thus the homogeneity of the plasma and optical thinness are assumed, that is, the reabsorption effect is neglected for the plasma induced on the liquid surface.

(5) The laser-induced plasma is treated as a closed system, where matter conservation and electrical neutrality are assumed. Of course this assumption is suspect but it is made in this work to simplify the problem.

2.2. LTE-Model. Considering the above assumptions, the intensity $I_{m l}$ of given spectral line emitted from the laserinduced plasma, corresponding to a transition from an upper excited level $m$ to a lower excited level $l$ is given by $[15,16]$

$$
I_{m l}=F \cdot A_{m l} n_{m} \cdot h v_{m l} \text {, }
$$

$F$ is an experimental factor which depends on the probing volume of the plasma and the spectral response of the detection system, $A_{m l}$ is the transition probability from level $m$ to $l, n_{m}$ is the number density of atoms on the upper excited level $m, h$ is the Planck's constant, and $v_{m l}$ is the frequency of the transition.

As the laser-induced plasma satisfied the local thermodynamic equilibrium (LTE), levels populations obey the Boltzmann statistic, that is, the population $n_{m}(T)$ of an upper excited level $m$ is related to the total number density $n(T)$ by the Boltzmann equation [18]:

$$
n_{m}(T)=\frac{n(T)}{U(T)} \cdot g_{m} \exp \left(-\frac{E_{m}}{k T}\right),
$$

$g_{m}$ is the statistical weight of the excited level $m, E_{m}$ is the corresponding energy, and $k$ is Boltzmann's constant.

$U(T)$ is the partition function of the corresponding specie, its value, is well tabulated in $[19,21]$.

In this work, the partition functions have been computed using NIST energy level data [21]; then the obtained values are verified with those reported by Drawin and Felenbok [19].

It should be noted that the relevant partition functions are those calculated by using only observed levels. This is a crude approximation because it neglects the existence of many other levels. However, in general, partition function varied slightly over low-temperature range.

According to (1), to understand the dependence of line intensity on the plasma temperature, the evolution of excited level population $n_{m}(T)$ as function of temperature should be investigated, which require the determination of the corresponding specie density $n(T)$, as shown in (2).

However, assuming the LTE state of the laser-induced plasma, a system of equations with species densities as unknowns could be formed by Dalton, matter conservation, and Saha and Guldberg-Waage equations. This equilibrium model based on the LTE assumption is referred to, as the LTEmodel.

\section{Results and Discussion}

3.1. Plasma Composition. Adopting the LTE-model, a code is written in Maple language for the numerical computation of the plasma composition. The "solve" Maple command is used for solving the system of equations. 


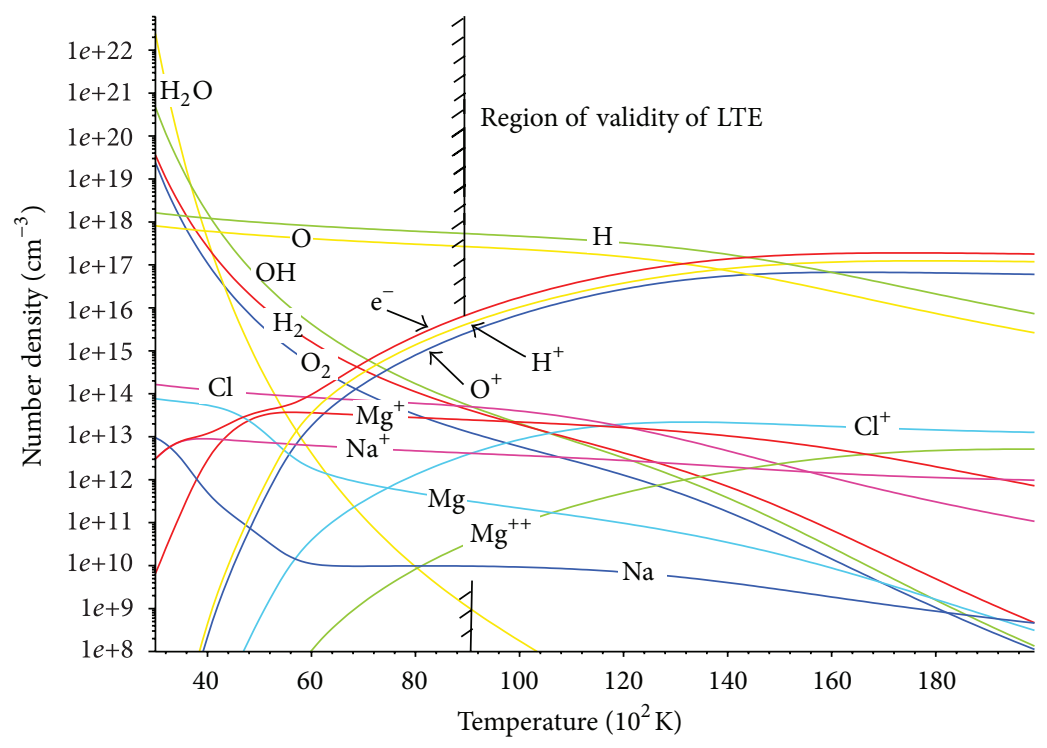

Figure 1: Species densities as function of temperature, for plasma of $\mathrm{MgCl}_{2}-\mathrm{NaCl}$ solution concentrated at $50 \mathrm{mg} / \mathrm{L}$, at atmospheric pressure under thermal equilibrium

The system is initialized by an approached value of the electron density at the starting calculation point, then equations system is solved and a new value for $n_{e}$ is calculated.

This code has been adapted to calculate the composition of equilibrium plasma of $50 \mathrm{mg} / \mathrm{L} \mathrm{MgCl}_{2}-\mathrm{NaCl}$ aqueous solution at atmospheric pressure, for given temperature.

To corroborate our calculation, species number densities calculated with this numerical code for water-alkaline salt plasma are compared with previous published data in [17], the model reproduces with satisfactory accuracy results reported by Hannachi et al. It is also interesting to make comparison with experimental data from literature, but to our knowledge, there are no available experimental measurements for species number densities of plasma induced in aqueous solution.

Results presented in Figure 1 illustrate densities of elemental species considered in the equilibrium plasma of $50 \mathrm{mg} / \mathrm{L} \mathrm{MgCl}_{2}-\mathrm{NaCl}$ aqueous solution at atmospheric pressure, for temperatures ranging from 3000 to $20000 \mathrm{~K}$.

Figure 1 shows a progressive disappearance of neutral species with increased temperature. In parallel, densities of charged species $\left(\mathrm{H}^{+}, \mathrm{O}^{+}\right.$, and $\left.\mathrm{Cl}^{+}\right)$grow up exponentially; for example, density of $\mathrm{H}^{+}$ion, $n_{\mathrm{H}^{+}}(T)$ gain two orders of magnitudes from $6000 \mathrm{~K}$ to $12000 \mathrm{~K}$. In this temperature range, the intensive ionization of major species leads to an increase of the electron density; this excess of electron density favors the recombination process of $\mathrm{Na}^{+}$ions, due to the low-ionization potential of sodium. This process of $\mathrm{Na}^{+}$ recombination compensate the sodium ionization process, which explain the flat curve of sodium density noted between $6000 \mathrm{~K}$ and $12000 \mathrm{~K}$.

After reaching maximal values, charged species densities follow a slight decrease, for example, $\mathrm{H}^{+}$density stays nearly constant around $17000 \mathrm{~K}$, with $n_{\mathrm{H}^{+}}(T) \sim 10^{17} \mathrm{~cm}^{-3}$, then it decrease slightly from $19000 \mathrm{~K}$. This weak decrease is interpreted with the Dalton's law. Since the total pressure of the plasma is supposed to be invariable, the temperature increase must be accompanied with a reduction of the total number of species which promote recombination process, and consequently decrease the charged species density.

Concerning the electrons density $n_{e}$, three regions can be distinguished. At low plasma temperature $(T<4000 \mathrm{~K})$, the electrons density can be identified by $\mathrm{Na}^{+}$density: $n_{e} \sim$ $n_{\mathrm{Na}^{+}}$; indeed, sodium presents the lowest ionization energy $(5.1 \mathrm{eV})$, thus, for low temperature the sodium ionization is nearly the sole source of electrons. In the temperature range $4000-5000 \mathrm{~K}$, the neutrality is affected by the production of the $\mathrm{Mg}^{+}$ions, and the evolution of $n_{e}$ follow the evolution of $\mathrm{Mg}^{+}$density. For temperature higher than $5000 \mathrm{~K}, n_{e}$ keeps a profile intimately adjacent to $n_{\mathrm{H}^{+}}$; the neutrality is assured by hydrogen ion $\mathrm{H}^{+}$; this is evident, since hydrogen, which has the highest ionization energy $(13.6 \mathrm{eV})$, is the major element in the solution. At $17000 \mathrm{~K}$, the plasma reaches the saturation of electrons, with electron number density around $10^{17} \mathrm{~cm}^{-3}$. Beyond $17000 \mathrm{~K}$, the electron density undergoes a slight decrease. In fact, at such elevated temperature the plasma is saturated of ionized species, and the electron density $n_{e}$ reaches higher values; therefore, Le Chatelier's principle suggests that equilibrium is displaced towards the reduction of those species by recombination process.

Figure 1 shows the region of validity of the LTE assumption. In fact, the lower limit of electron density for which the plasma satisfies the LTE condition has been estimated to $10^{16} \mathrm{~cm}^{-3}[9,10]$. According to Figure 1, the region of validity of LTE corresponds with temperature higher than $9000^{\circ} \mathrm{K}$. 
TABle 1: Atomic data of MgII and NaI lines [22].

\begin{tabular}{lccccccc}
\hline Element & $\lambda(\mathrm{nm})$ & Transition & $E_{1}(\mathrm{eV})$ & $E_{2}(\mathrm{eV})$ & $A_{21}\left(10^{8} \mathrm{~s}^{-1}\right)$ & $g_{1}$ & $g_{2}$ \\
\hline $\mathrm{Mg}$ & 279.55 & $3 \mathrm{~s}^{2} \mathrm{~S}-3 \mathrm{p}^{2} \mathrm{P}$ & 0 & 4.43 & 2.68 & 4 \\
$\mathrm{Na}$ & 588.99 & $3 \mathrm{~s}^{2} \mathrm{~S}-3 \mathrm{p}^{2} \mathrm{P}$ & 0 & 2.104 & 6.28 & 2 \\
\hline
\end{tabular}

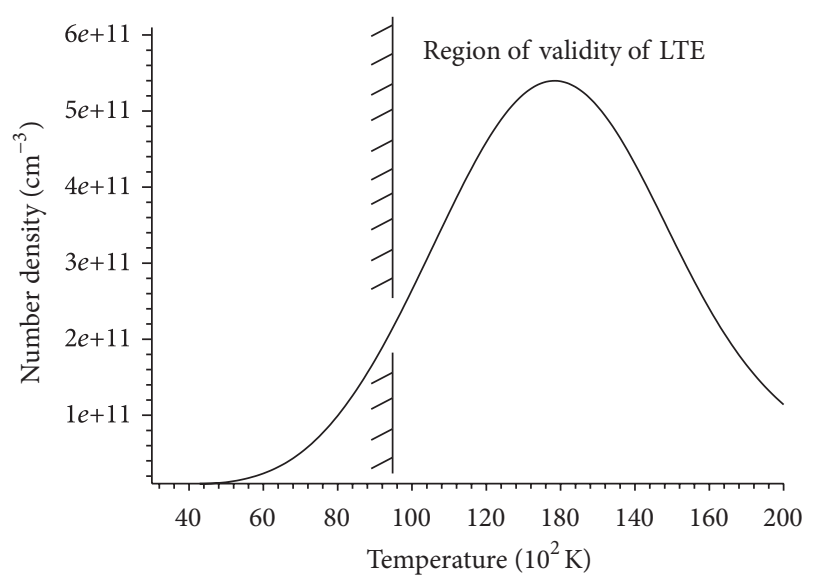

Figure 2: Population of excited level $\operatorname{Mg}(3 p)$, as function of temperature, in the equilibrium plasma of $\mathrm{MgCl}_{2}-\mathrm{NaCl}$ solution concentrated at $50 \mathrm{mg} / \mathrm{L}$.

3.2. Number Densities of Emitting Species. The spectroscopic analysis of LIBS spectrum obtained from water sample shows that only resonance emission lines, corresponding with lower energy excited states, exhibit well-resolved lines $[5,9]$. This is due to the rapid water-plasma cooling; in fact plasma emission lifetimes are usually very short under such hydrated environment.

The most intense resonance lines, recorded in the LIBS spectrum of the $\mathrm{MgCl}_{2}-\mathrm{NaCl}$ aqueous solution, are MgII $(279 \mathrm{~nm})$ and $\mathrm{NaI}(588 \mathrm{~nm})$ lines [9]. Table 1 provides the energy levels, transition probabilities, and electronic configuration of those resonance lines.

Table 1 shows that MgII and NaI lines are emitted, respectively, from the magnesium-ionized species in the $\mathrm{Mg}(3 \mathrm{p})$ excited state, and the sodium neutral specie in its $\mathrm{Na}(3 \mathrm{p})$ excited state.

Applying the Boltzmann law equation (2), densities of those emitting excited species have been calculated from total densities of corresponding species which are already obtained by the LTE-model.

3.2.1. Population of Excited Level $M g(3 p)$. Figure 2 present the emitting-atom densities at the excited level $\mathrm{Mg}(3 \mathrm{p})$ of the spectral lines $\operatorname{MgII}(279 \mathrm{~nm})$, as function of temperature, at atmospheric pressure in the plasma of $\mathrm{MgCl}_{2}-\mathrm{NaCl}$ solution concentrated at $50 \mathrm{mg} / \mathrm{L}$.

This figure shows that MgII spectral line reaches a maximum of upper-level population, at so-called norm temperature, $T^{*}[18,23]$, assessed to $13800 \mathrm{~K}$. The notion of norm temperature explains the competition between two

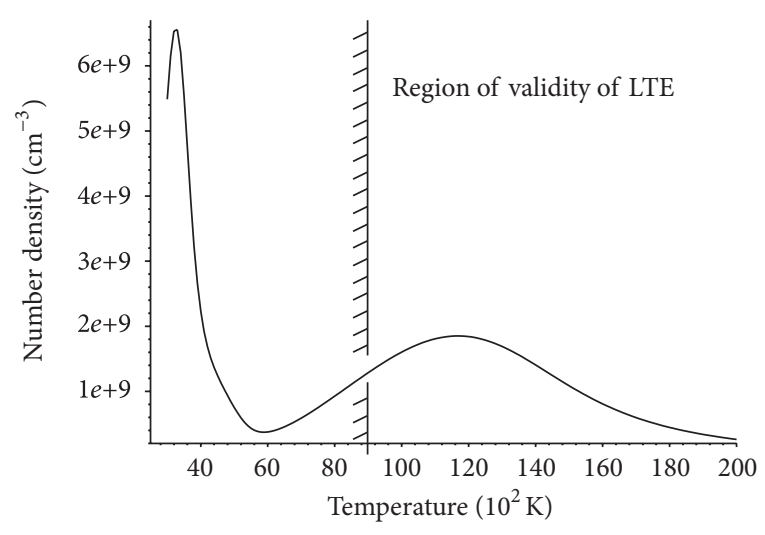

FIgURE 3: Population of excited level $\mathrm{Na}(3 \mathrm{p})$, as function of temperature, in the equilibrium plasma of $\mathrm{MgCl}_{2}-\mathrm{NaCl}$ solution concentrated at $50 \mathrm{mg} / \mathrm{L}$.

processes promoted by the increase of temperature, namely, the excitation and the ionization. The excitation is a factor of population, contrarily, the ionization constitutes a factor of impoverishment for the emitting level.

A more detailed explanation is given by Boltzmann equation Equation (2) shows that the number of atoms on the superior level $n_{m}(T)$ is essentially a product of two temperature functions, $n(T)$ and $\exp \left(-E_{k}, / k T\right)$. The partition function varies slightly at the considered range temperature. For low temperatures, the ionization process is so weak, thus the total atoms density $n(T)$ kept a constant value, and by the steep increase of the exponential function, the number of atoms in the superior level increases with the temperature. For high temperatures, the exponential term approaches unity; but, owing to the intensification of ionization process, the total atoms density $n(T)$ decrease with the temperature and it follows a reduction of atoms on the superior level. This change of emitting-atoms densities trend shows that for a spectral line, there is a maximum of population at a certain temperature $T^{*}$.

3.2.2. Population of Excited Level $\mathrm{Na}(3 p)$. Figure 3 presents the number density on the emitting level $\mathrm{Na}(3 \mathrm{p})$ of the sodium line $\mathrm{NaI}$, as function of plasma temperature, at atmospheric pressure in the plasma of $\mathrm{MgCl}_{2}-\mathrm{NaCl}$ solution concentrated at $50 \mathrm{mg} / \mathrm{L}$.

Unlike the MgII spectral line, NaI line presents two maxima of upper-level population, at norm temperatures of 3300 and $11700 \mathrm{~K}$. It should be noted that NaI norm temperatures are both lower than that of MgII. 
The curve of number density of $\mathrm{Na}(3 \mathrm{p})$-excited atoms as a function of temperature (Figure 3), can be interpreted as follows.

At low temperature $(T<3300 \mathrm{~K})$, the increase of plasma temperature will serve to populate the excited level (as predicted by the exponential term of the Boltzmann equation). For temperatures ranging from 3300 to $5900 \mathrm{~K}$, the ionization process of sodium becomes dominant and thus, there will be a decrease of sodium density and subsequently the density of sodium atoms on exited state $\mathrm{Na}(3 p)$ decreases in this temperature range. In the temperature range 5900-11700 K, the ionization of major species in the aqueous solution, specifically hydrogen and oxygen atoms, leads to the increase of the electron density (Figure 1), this overload of electrons in the plasma favors the recombination process of $\mathrm{Na}^{+}$ ions. This explains why the recombination and ionization processes are almost compensated in this temperature range, that is, the sodium density stay nearly constant as shown in Figure 1. As sodium density is roughly invariant in this temperature range (5900-11700 K), owing to the exponential term of the Boltzmann equation, the population of $\mathrm{Na}(3 \mathrm{p})$ increases with temperature. For temperature higher than $11700 \mathrm{~K}$ the ionization dominates again, so the sodium atom density including excited-sodium density decreases with temperature.

Summing up, it seems that, the appearance of two norm temperatures for the $\mathrm{NaI}$ line is due, on the one hand to the near to the ground correspondent $\mathrm{NaI}$ emitting level and the low-ionization potential of the sodium atom and on the other hand to the low concentration of this species in the aqueous solution.

To make in evidence the effect of sodium concentration on the NaI norm temperatures, the density of excited sodium at $\mathrm{Na}(3 p)$ state, as function of temperature is represented in Figure 4, for different concentration of sodium in the $\mathrm{MgCl}_{2}$ $\mathrm{NaCl}$ aqueous solution.

This figure displays a disappearance of the splitting of $\mathrm{NaI}$ norm temperature for higher concentration of sodium. As $\mathrm{Na}$ concentration increase, the first maximum of $\mathrm{NaI}$ upper level population approaches the second one till both overlap forming only one maximum. The second norm temperature is approximately the same for all aqueous solutions. In fact the second peak of the density of excited sodium is related to the ionization of major species in the aqueous solution, such as hydrogen and oxygen atoms.

\section{Conclusion}

This work presents a theoretical interpretation for the understanding of the plasma temperature effect on the spectral emission of plasma induced in $\mathrm{MgCl}_{2}-\mathrm{NaCl}$ aqueous solution. LTE model is carried out to compute the plasma composition and it allowed the estimation of atoms, ions, and electron densities in the laser-induced plasma in the water sample at atmospheric pressure, for temperature ranging from $3000 \mathrm{~K}$ to $20000 \mathrm{~K}$. The populations of excited species emitting MgII and $\mathrm{NaI}$ lines are also calculated.

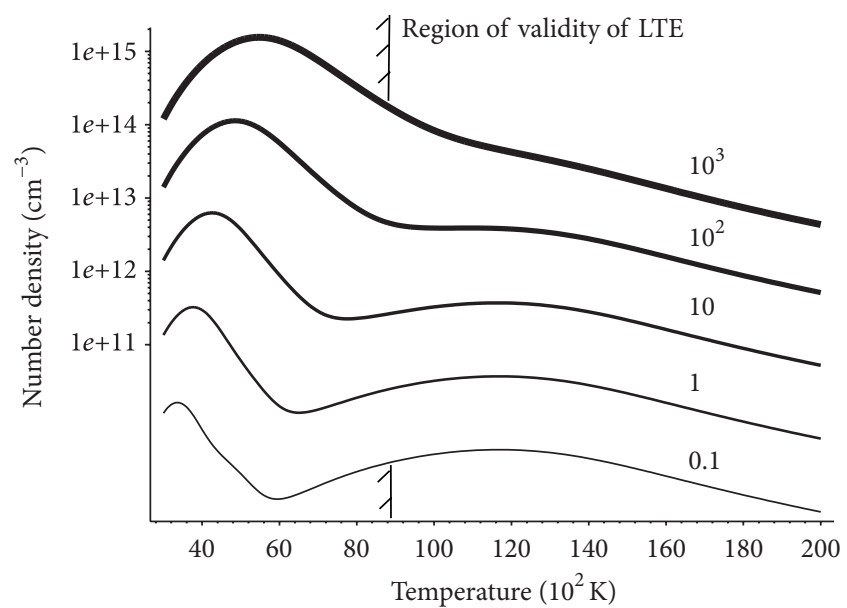

FIGURE 4: Density of sodium at $\mathrm{Na}(3 \mathrm{p})$-excited states, as function of temperature, in equilibrium plasma induced on $\mathrm{MgCl}_{2}-\mathrm{NaCl}$ aqueous solution, for different sodium concentration (shown in $\left.\mathrm{g} \cdot \mathrm{L}^{-1}\right)$

The obtained results reveal that MgII line presented a maximum of population on the issuing level, at norm temperature of $13800 \mathrm{~K}$; However, NaI line presents two norm temperatures both lower than the MgII norm temperature. Considering the optically thinness assumption of the laser-induced plasma, the intensity of given spectral line is proportional to the number density of atoms on the upper excited level. Therefore, for better sensibility of the LIBS technique, comparing to the $\mathrm{Mg}$ II line, it is recommended to record $\mathrm{NaI}$ signal at relatively cold plasma, either by lowering the laser pulse energy, or by detecting the spectral emission at late stage of the plasma lifetime. Moreover, when using common broadband spectrometer systems that measure both lines at once it is suggested that spectrometers should collect light from early in the plasma when it is hot and stay open until it is cold to maximize signal from these two elements.

It should be noted that the numerical model built in this study is not restricted to magnesium and sodium aqueous solutions; it could be applied to any other water-alkaline salt mixture. As seen for magnesium and sodium species, for any other alkaline species such as lithium, calcium, and potassium, the corresponding norm temperature will depend essentially on the ionization potential, upper energy level, and dominance of the considered alkaline species in the aqueous solution.

In this work, it is assumed that the laser-induced plasma is ideal, that is, spatially homogeneous, electrically neutral, optically thin, in local thermodynamic equilibrium (LTE). In reality, not all these conditions may be fulfilled; the effect of departures from plasma ideality is planned for future investigations.

\section{References}

[1] L. J. Radziemski and D. A. Cremers, Laser-Induced Plasmas and Applications, Marcel Dekker, New York, NY, USA, 1989. 
[2] M. Sabsabi and R. Russo, "Fourth international conference on laser induced plasma spectroscopy and applications LIBS 2006 Montreal-Canada," Spectrochimica Acta Part B, vol. 62, no. 12, pp. 1285-1286, 2007.

[3] P. Yaroshchyk, D. Body, R. J. S. Morrison, and B. L. Chadwick, "A semi-quantitative standard-less analysis method for laserinduced breakdown spectroscopy," Spectrochimica Acta Part B, vol. 61, no. 2, pp. 200-209, 2006.

[4] D. A. Cremers, L. J. Radziemski, and T. R. Loree, "Spectrochemical analysis of liquids using the laser spark," Applied Spectroscopy, vol. 38, no. 5, pp. 721-729, 1984.

[5] O. Samek, D. C. S. Beddows, J. Kaiser et al., "Application of laser-induced breakdown spectroscopy to in situ analysis of liquid samples," Optical Engineering, vol. 39, no. 8, pp. 2248-2262, 2000.

[6] V. Lazic, F. Colao, R. Fantoni, and V. Spizzicchino, "Laserinduced breakdown spectroscopy in water: improvement of the detection threshold by signal processing," Spectrochimica Acta Part B, vol. 60, no. 7-8, pp. 1002-1013, 2005.

[7] L. St-Onge, E. Kwong, M. Sabsabi, and E. B. Vadas, "Rapid analysis of liquid formulations containing sodium chloride using laser-induced breakdown spectroscopy," Journal of Pharmaceutical and Biomedical Analysis, vol. 36, no. 2, pp. 277-284, 2004.

[8] A. De Giacomo, M. Dellaglio, F. Colao, R. Fantoni, and V. Lazic, "Double-pulse LIBS in bulk water and on submerged bronze samples," Applied Surface Science, vol. 247, no. 1-4, pp. 157-162, 2005.

[9] B. Charfi and M. A. Harith, "Panoramic laser-induced breakdown spectrometry of water," Spectrochimica Actan Part B, vol. 57, no. 7, pp. 1141-1153, 2002.

[10] M. Adamson, A. Padmanabhan, G. J. Godfrey, and S. J. Rehse, "Laser-induced breakdown spectroscopy at a water/gas interface: a study of bath gas-dependent molecular species," Spectrochimica Acta Part B, vol. 62, no. 12, pp. 1348-1360, 2007.

[11] J. B. Ahmed and N. Jaïdane, "Temporal behavior of neutral and ionic lines emitted from a laser induced plasma on an aqueous surface," Spectrochimica Acta Part B, vol. 64, no. 5, pp. 442-447, 2009.

[12] R. Fantoni, L. Caneve, F. Colao, L. Fornarini, V. Lazic, and V. Spizzichino, "Methodologies for laboratory Laser induced breakdown spectroscopy semi-quantitative and quantitative analysis," Spectrochimica Acta Part B, vol. 63, no. 10, pp. 1097-1108, 2008.

[13] A. R. Casavola, G. Colonna, and M. Capitelli, "Kinetic model of titanium laser induced plasma expansion in nitrogen environment," Plasma Sources Science and Technology, vol. 18, no. 2, Article ID 025027, 8 pages, 2009.

[14] M. Capitelli, F. Capitelli, and A. Eletskii, "Non-equilibrium and equilibrium problems in laser-induced plasmas," Spectrochimica Acta Part B, vol. 55, no. 6, pp. 559-574, 2000.

[15] C. Aragón and J. A. Aguilera, "Characterization of laser induced plasmas by optical emission spectroscopy: a review of experiments and methods," Spectrochimica Acta Part B, vol. 63, no. 9, pp. 893-916, 2008.

[16] M. A. Ismail, G. Cristoforetti, S. Legnaioli et al., "Comparison of detection limits, for two metallic matrices, of laser-induced breakdown spectroscopy in the single and double-pulse configurations," Analytical and Bioanalytical Chemistry, vol. 385, no. 2, pp. 316-325, 2006.

[17] R. Hannachi, Y. Cressault, P. Teulet, Z. Ben Lakhdar, and A. Gleizes, "Net emission of $\mathrm{H}_{2} \mathrm{O}-$ air $-\mathrm{MgCl}_{2} / \mathrm{CaCl}_{2} / \mathrm{NaCl}$ thermal plasmas," Journal of Physics D, vol. 41, no. 20, Article ID 205212, 12 pages, 2008.

[18] W. Lochte-Holtgreven, Plasma Diagnostics, AIP press, New York, NY, USA, 1995.

[19] H. W. Drawin and P. Felenbok, Data for Plasmas in Local Thermodynamic Equilibrium, Gauthier-Villars, Paris, Farnce, 1965.

[20] H. R. Griem, Plasma Spectroscopy, McGraw-Hill, New York, NY, USA, 1964.

[21] National Institute of Standards and Technology, NIST electronic database, http://physics.nist.gov/PhysRefData/ASD/ lines_form.html.

[22] S. Megy, J. M. Baronnet, and E. A. Pavlov, "Distribution of plasma parameters in a nitrogen transferred DC arc," Journal of Physics D, vol. 28, no. 2, pp. 344-352, 1995.

[23] J. W. Olesik, "Consideration of norm temperature and local thermodynamic equilibrium models for emission and ionization in inductively coupled plasmas," Spectrochimica Acta Part $B$, vol. 44, no. 6, pp. 625-638, 1989. 

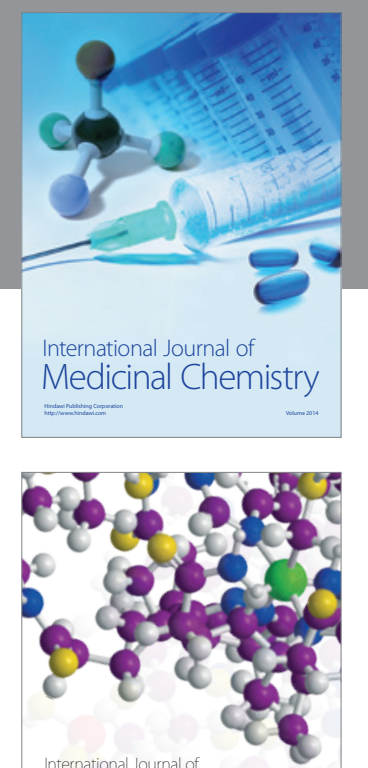

\section{Carbohydrate} Chemistry

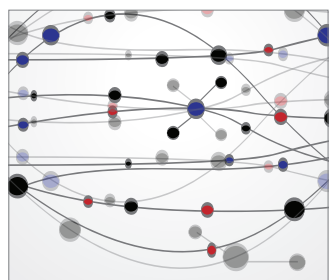

The Scientific World Journal
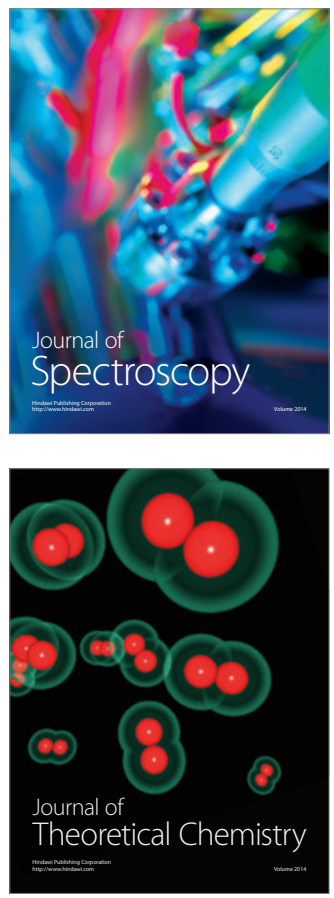
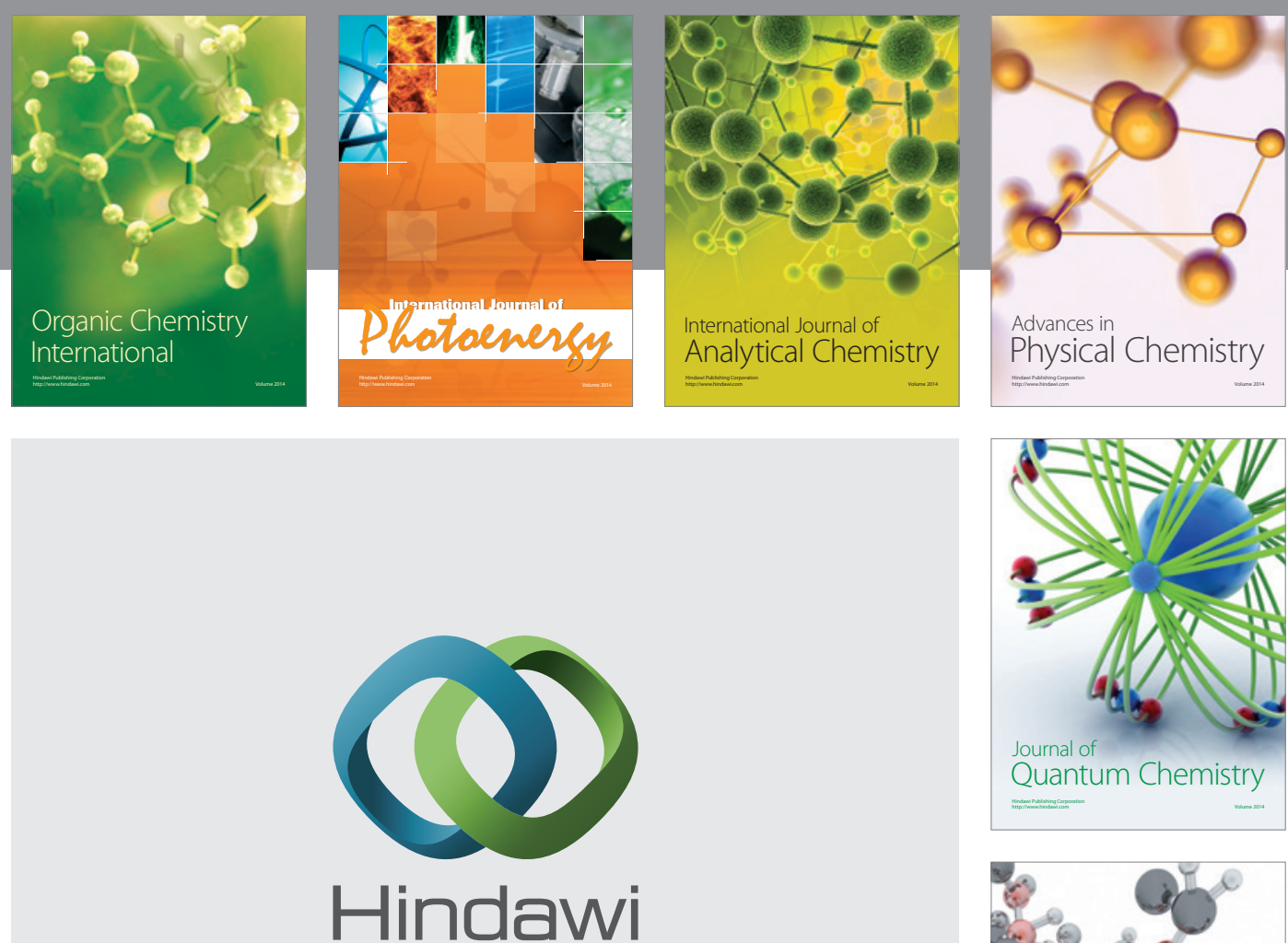

Submit your manuscripts at

http://www.hindawi.com

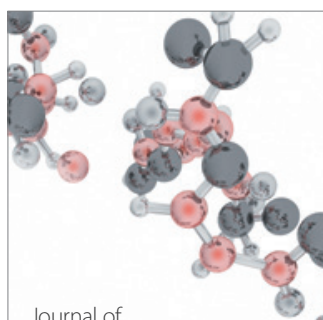

Analytical Methods

in Chemistry

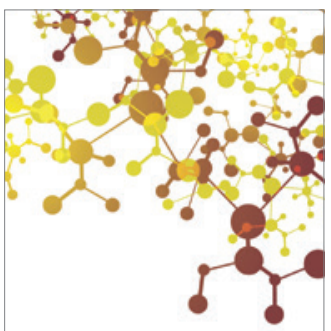

Journal of

Applied Chemistry

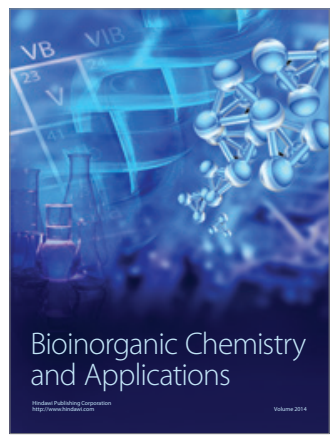

Inorganic Chemistry
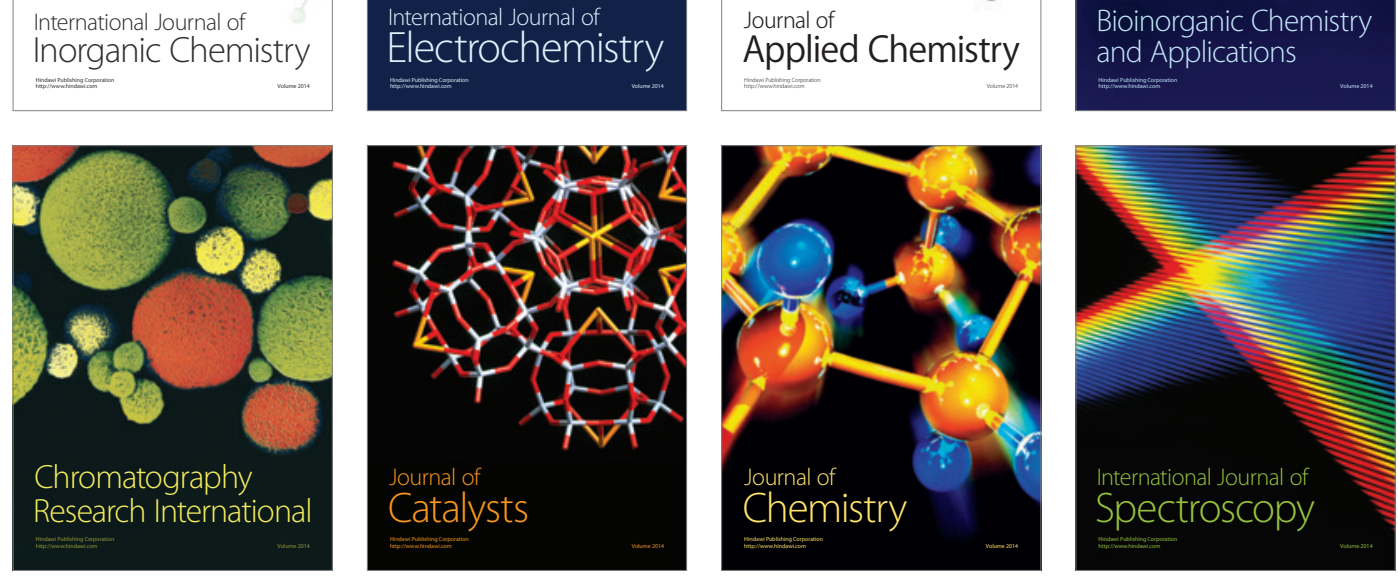